\title{
LOS GLACIARES ROCOSOS DE LA SIERRA DEL SUSPIRÓN (LEÓN)
}

\author{
Alipio GARCÍA DE CELIS \\ Departamento de Geografía, Universidad de León
}

\section{LAS CLAVES LITOESTRUCTURALES DE LA SIERRA DEL SUSPIRÓN}

La Sierra del Suspirón es una alineación montañosa de orientación ONOESE que se extiende desde el valle del río Boeza hasta la cabecera del río Negro, en una longitud de unos quince kilómetros. En esta misma dirección presenta una altitud decreciente, desde los $2.058 \mathrm{~m}$. del pico «Arcos del Agua» hasta los 1.638 del monte «Prado Redondo».

Dada la adaptacion de la sierra a las directrices marcadas por la megaestructura de la rodilla astúrica, los materiales se disponen en bandas longitudinales, orientadas de NO. a SE. Geológicamente pertenecen al Cámbrico y Ordovícico, desde la Formación Cándana-Herrería (Cámbrico inferior), que contacta por el N. mediante falla con el Precámbrico del núcleo del Antiforme del Narcea, hasta las pizarras de Luarca (Ordovícico inferiorsuperior), que a través de otras fracturas entran en contacto por el S. con el Carbonífero de la cuenca del Bierzo. No obstante, son las cuarcitas, areniscas y pizarras de la potente Serie de Los Cabos las que forman el cuerpo central de la Sierra, en una anchura de entre tres y cuatro kilometros.

Estos materiales aparecen, debido a la orogenia herciniana, con buzamiento vertical o subvertical y con un diaclasamiento muy denso, hecho de gran trascendencia en la explicación de la génesis de los modelados tardiglaciares (GUTIÉRREZ EloRZA y PEÑA MONNE, 1981: 107). Asimismo, la alternancia de estratos de cuarcitas, areniscas y pizarras de poco espesor en esta serie, con predominio de las cuarcitas, tiene gran importancia en el modelado de la Sierra, al ofrecer abundante material de gelifracción allí donde los estratos forman escarpes, y porque la presencia de capas de diferente dureza y crestas estructurales ha facilitado el desarrollo de paredes de circos, umbrales y fenómenos de sobreexcavación glaciar.

\section{CARACTERES GENERALES DEL MODELADO GLACIAR DE LA SIERRA}

Durante la fase del máximo glaciar se desarrollaron un total de 17 aparatos glaciares en la $\mathrm{S}^{\mathrm{a}}$. del Suspirón, incluyendo tanto lenguas de más de un kilómetro de longitud como pequeños glaciares de circo. De estos diecisiete aparatos, diez corresponden a la vertiente septentrional y siete a la meridio- 
nal (ver Fig. 1).

Por lo que respecta a las áreas de acumulación, se trata en la mayoría de los casos de circos «de cubeta» ${ }^{1}$, con sus fondos situados entre $1.450 \mathrm{y}$ $1.850 \mathrm{~m}$. de altitud y orientados mayoritariamente al NE.

Son casi todos aparatos individualizados, que muerden la cabecera de antiguos valles, sobreexcavándoles y modelándoles en artesa aguas abajo, pues también casi todos ellos dieron lugar a pequeñas lenguas glaciares que en algún caso, como ya hemos señalado, superaron el Km. de longitud.

Las áreas de descarga son normalmente difíciles de establecer, pues en casi todos los aparatos glaciares se aprecian dos pulsaciones, una de máximo avance, cuyos depósitos morrénicos, desaparecidos o muy destruídos por el modelado posterior, parecen situarse a altitudes de entre 1.290 y $1420 \mathrm{~m}$. Otra en pleno proceso de retroceso, que dejó nítidos depósitos por encima de $1.400-1.600 \mathrm{~m}$.

Las pequeñas dimensiones de los aparatos, el carácter aislado de unos con respecto a otros, su adaptación a topografías preexistentes y la disimetría en el modelado glaciar de una vertiente con respecto a la otra, nos indica que se trata de un modelado glaciar marginal, desarrollado bajo unas condiciones límite y que no supuso más que un retoque en la morfología de la Sierra. Sin embargo, y quizá por la relativamente escasa personalidad del modelado glaciar, los fenómenos de modelado ligados a un glaciarismo tardío adquieren especial relevancia, pues adaptándose a unas condiciones litológicas especialmente favorables, desarrollaron en la parte más elevada de la Sierra formas de grandes dimensiones, riqueza de matices y calidad de conservación prácticamente únicas en todo el conjunto de la Montaña Occidental de León.

\section{LAS FORMAS DE RELIEVE LIGADAS A LA FASE TARDIGLACIAR EN EL SECTOR CULMINANTE DE LA SIERRA DEL SUSPIRÓN}

Aparecen formas tardiglaciares en áreas más bajas de la Sierra, en forma de guirnaldas o incipientes morrenas de nevero. Es el caso de la que se desarrolla sobre la pared orientada al N. del circo de «Rairisa» (no 2 en la Fig. 1 ), en el valle del mismo nombre y a $1.650 \mathrm{~m}$. de altitud. Es una guirnalda en forma de arco, de $100 \mathrm{~m}$. de longitud y entre 20 y 50 de anchura, con unos $10 \mathrm{~m}$. de altura en el frente, aunque esta altura resulta difícil de establecer, dada la fuerte pendiente de la ladera sobre la que se asienta.

No obstante, como ya se ha señalado, las formas tardiglaciares mejor desarrolladas se circunscriben al sector culminante de la sierra, lo que podríamos denominar como «Macizo de Arcos del Agua». En este sector se desarrollaron las cabeceras de cuatro aparatos glaciares, dos en la vertiente

${ }^{1}$ Seguimos la tipología establecida por Tricart y Cailleux (TRICART y CAILLEUX, 1962, pp. 216 y ss.). 
N. -los más importantes de toda la sierra, con lenguas que superaron los $1.500 \mathrm{~m}$. de longitud- y otros dos en la S. -reducidos a glaciares de circo, uno de ellos con una pequeña lengua de prolongación_ ( $\mathrm{n}^{\circ} 10,11,13$ y 14 en la Fig. 1).

Sobre este área glaciada, en la fase final del retroceso glaciar, se establecen dos glaciares rocosos y un nevero, aprovechando probablemente las últimas manchas de hielo glaciar.

\subsection{El nevero de «Peña Cefera»}

Sobre la pared orientada al N. del circo glaciar de Arcos del Agua (n⿳0 11 en la Fig. 1), permaneció un nevero que dió lugar a una potente acumulación morrénica a sus pies. Esta morrena presenta forma de arco (ver Fig. 2 y Fig. 5), con unos $250 \mathrm{~m}$. de longitud y entre 5 y $15 \mathrm{~m}$. de anchura. En la zona central, más potente, la morrena destaca del terreno en la actualidad unos 5-10 m. La altitud a la que se sitúa está en torno a los 1.915-1.960 m., mientras que la cresta rocosa de la que procedía el material (la pared $\mathrm{N}$. del pico «Peña Cefera») alcanza los $2.010 \mathrm{~m}$.

El material que forma la morrena está constituído por bloques de cuarcita, angulosos, de fuerte heterometría. Algunos de ellos, sobre la morrena, alcanzan los $10 \mathrm{~m}^{3}$ de volumen. Hay una total ausencia de material fino.

El frente de la morrena no es muy abrupto, aunque esto puede deberse a una removilización del material posterior a su deposición, en parte por la dinámica periglaciar y en parte por la propia fusión del nevero.

Algo similar ocurre con la pared del nevero, cuya pendiente, normalmente no inferior a $45^{\circ}-50^{\circ}$, es en la actualidad de $35^{\circ}-40^{\circ}$, es decir, se ha suavizado como consecuencia del recubrimiento que presenta en forma de canchales. Estos canchales presentan menor heterometría, especialmente destaca la ausencia de grandes bloques.

En la cresta que limita la parte superior del nevero, los estratos de cuarcita afloran con buzamientos verticales y rumbo O.-E., con lo cual la pared del nevero - la antigua pared del circo glaciar- en unos tramos corta los estratos a bisel y en otros se adapta a sus planos de estratificación; todo ello facilitó enormemente la actuación de la dinámica hielo-deshielo y el desprendimiento de grandes cantidades de material.

Este detalle, unido a la orientación de la pared y a la altitud, explica la formación de una morrena de nevero tan perfecta.

Las diferencias con las morrenas glaciares de retroceso situadas sobre la misma plataforma del circo glaciar (ver Fig. 5) son nítidas. Mientras que éstas aparecen recubiertas por un tapiz herbáceo e incluso un matorral denso y contínuo de leguminosas, la morrena del nevero presenta una exposición subaérea limpia, sus bloques están colonizados sólamente por líquenes, existiendo muy poca vegetación de porte herbáceo o arbustivo.

Esto se explica, a nuestro juicio, por la ausencia de material fino que rellene los huecos entre los bloques, algo propio, por el contrario, de las morrenas glaciares. En una morrena de nevero el material fino es muy esca- 
so o inexistente (TRICART y CAILlEUX, 1962: 226), por lo que el agua percola con extrema rapidez, dificultando sobremanera el desarrollo de un tapiz vegetal.

\subsection{El glaciar rocoso de «Arcos del Agua»}

En el mismo circo de Arcos del Agua ( $n^{2} 11$ en la Fig. 1), adosado a la pared orientada al ENE., se desarrollo, en las últimas fases de retroceso glaciar, un pequeño glaciar rocoso de unos $100-120 \mathrm{~m}$. de longitud por 50 m. de anchura (ver Fig. 3 y Fig. 5). Su altitud es similar a la de la morrena de nevero, alrededor de $1.910 \mathrm{~m}$. en el frente, y presenta una lengua doble, adaptada probablemente a las irregularidades del terreno (un posible umbral de cuarcitas más resistentes infrayacente).

El material está constituído por bloques heterométricos de cuarcita, con especial abundancia de grandes bloques de más de $10 \mathrm{~m} 3$. de volumen.

Su topografía es muy irregular, debido a las reducidas dimensiones y a su adaptación al terreno, a consecuencia de lo cual las típicas ondas de flujo o cordones son discontínuas y poco nítidas, solapándose unas a otras. Se agrupan en dos conjuntos: en el frente del glaciar, un grupo de cuatro ondas, la principal del frente y otras tres menores situadas tras la primera, todas ellas orientadas al NE. Tras un escalón que parece deberse a la existencia de un umbral en el sustrato, y que divide al glaciar rocoso, una onda, de dimensiones similares a la frontal, se desvía ligeramente al ENE. e incluye en su interior a otras dos ondas de menores dimensiones, muy mal definidas.

Hacia la parte superior, el glaciar rocoso está fosilizado por los canchales desarrollados bajo la antigua pared del circo glaciar, en la que los estratos de cuarcita, verticales, aparecen cortados transversalmente e intensamente atacados por procesos de gelifracción.

La pared culmina a $2.060 \mathrm{~m}$., mientras que el glaciar arranca a $1.950 \mathrm{~m}$. Entre la culminación y los $2.000 \mathrm{~m}$. aproximadamente, la pared se muestra en su genuína verticalidad, mientras que entre los 2.000 y los $1.950 \mathrm{~m}$. los canchales periglaciares suavizan la pendiente y enlazan con el glaciar rocoso, fosilizándolo en parte.

El glaciar rocoso parece haberse desarrollado sobre los últimos restos de hielo glaciar de un antiguo aparato que, ocupando la amplia plataforma del circo, difluía hacia el N. y hacia el E., sobre el vecino valle de Peña Cefera. De sus últimas pulsaciones de avance y retroceso quedan varios arcos morrénicos (ver Fig. 5), el mayor de los cuales tiene su arranque casi imbricado con el frente del glaciar rocoso, diferenciándose ambos, no obstante, perfectamente, debido a las ostensibles diferencias en el recubrimiento vegetal.

De todo lo anteriormente dicho podemos concluir que se trata de un glaciar rocoso fósil, de tipo intermedio entre los glaciares rocosos en lengua (longitud mayor que anchura y cordones arqueados y superpuestos) y los glaciares rocosos lobados (anchura mayor que longitud y cordones coalescentes o imbricados lateralmente). Este carácter transicional o poco definido 

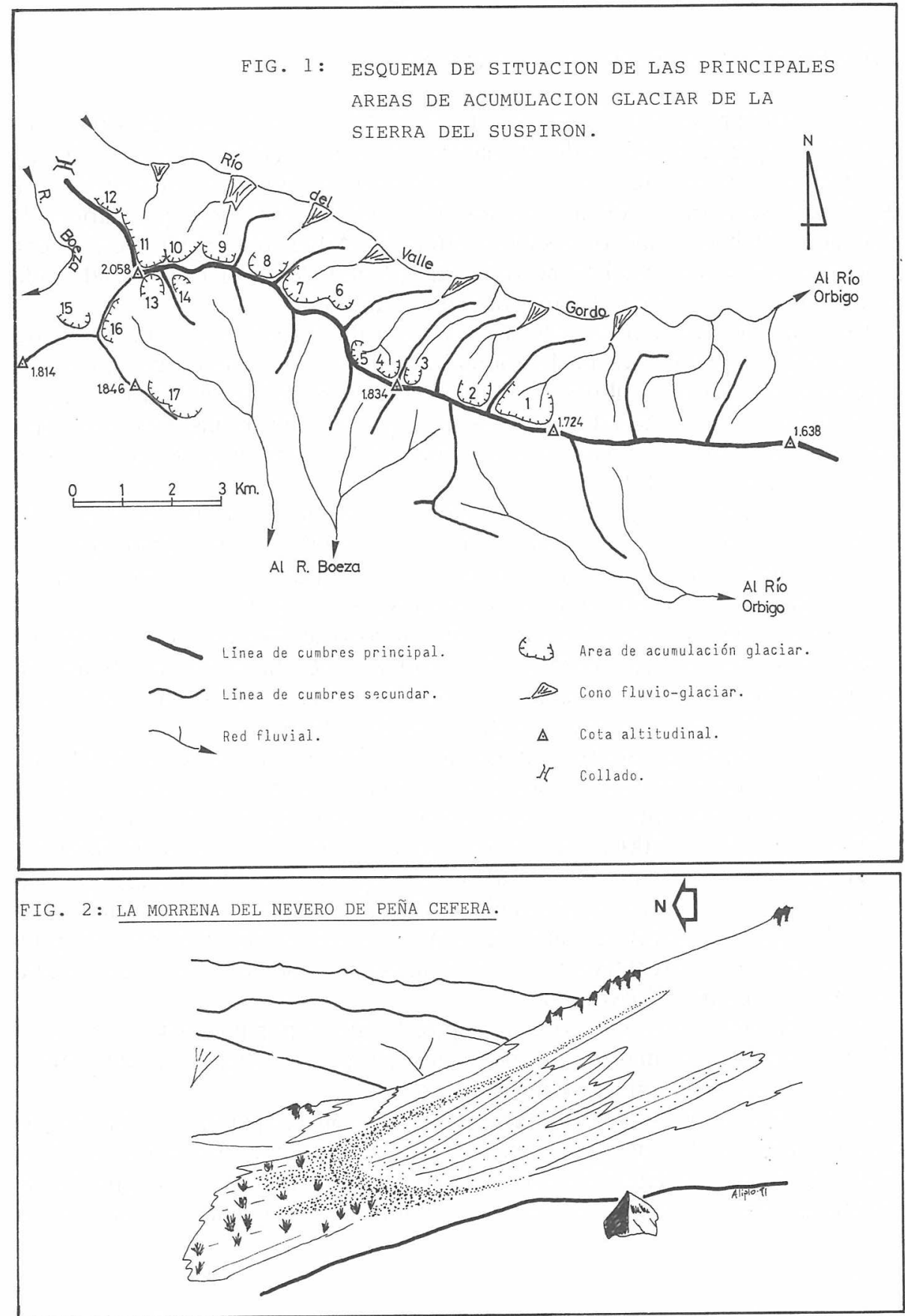
se deriva de sus pequeñas dimensiones y de su adaptación a una topografía irregular hecho que condiciona de forma principal la tipología de los glaciares rocosos (GóMEZ ORTIZ y LLOBET, 1988: 533).

\subsection{El glaciar rocoso de «Peña Cefera»}

El circo glaciar de Peña Cefera (no 10 en la Fig. 1) se desarrolla a una altitud menor que el de Arcos del Agua (el fondo de aquél se sitúa a $1.790 \mathrm{~m}$., mientras que el de éste está a $1.850 \mathrm{~m}$.) y entre ambos se establece un área de difluencia a través de un umbral. La sobrealimentación que supuso la afluencia de hielo glaciar desde el circo de Arcos del Agua facilitó una potente excavación en el lecho del valle glaciar de Peña Cefera, labrando una magnífica artesa en el duro roquedo cuarcítico.

En la última fase del periodo de retroceso de la masa de hielo, la disminuída lengua, privada ya de la sobrealimentación procedente de Arcös del Agua y adaptada al sombrío fondo de la artesa, se vió sepultada por el elevado volumen de material de gelifracción procedente de las abruptas paredes del circo, donde los estratos de cuarcita afloran verticales, en condiciones optimas de friabilidad.

De esta forma, mediante el empaste del hielo viejo con la masa de gelifractos procedente de las laderas, se formó el glaciar rocoso mejor desarrollado y conservado de toda la Montaña Occidental de León y, probablemente, de toda la Cordillera Cantábrica (ver Fig. 4 y Fig. 5).

En efecto, las dimensiones del glaciar rocoso de Peña Cefera no son en absoluto despreciables: $600 \mathrm{~m}$. de longitud y entre 100 y $120 \mathrm{~m}$. de anchu$\mathrm{ra}$, alcanzando la masa de derrubios una potencia en la zona central que estimamos que puede superar ampliamente los $30 \mathrm{~m}$.

Tiene su arranque aproximadamente a $1.760 \mathrm{~m}$. y muere a $1.600 \mathrm{~m}$., salvando por tanto un desnivel de $160 \mathrm{~m}$. con una pendiente del $26 \%\left(14^{\circ}\right)$. Se distinguen tres tramos principales; uno superior, entre los $1.760 \mathrm{~m}$. y un umbral situado a $1.700 \mathrm{~m}$. en el que se distinguen una onda principal y otras cinco menores, internas a aquélla. Un segundo tramo entre este umbral y la altitud de $1.620 \mathrm{~m}$. aproximadamente, marcado por una segunda gran onda de flujo que cobija en su interior a otras tres menores. Finalmente, un tercer tramo frontal formado por cuatro ondas de flujo peor definidas cuanto más nos acercamos al extremo.

El frente del glaciar rocoso es abrupto, formado por un caos de enormes bloques, superiores muchos de ellos a los $10 \mathrm{~m}^{3}$, con una altura de entre 5 y $10 \mathrm{~m}$. y una pendiente superior a $\operatorname{los} 20^{\circ}$.

El contacto de la lengua del glaciar rocoso con las paredes de la antigua artesa glaciar es limpio y neto en el caso de la pared orientada al ESE.; abundan en ella las superficies de roca pulida y las marcas del cepillado del primitivo glaciar ${ }^{2}$. Por el contrario, el contacto del glaciar rocoso con la

\footnotetext{
${ }^{2}$ La marca más destacable es una acanaladura de $6 \mathrm{~m}$. de longitud, $20 \mathrm{~cm}$. de anchura y
} 
pared de umbría, la que mira al ONO., está fosilizado por un extenso acúmulo de derrubios de gelifracción procedentes de la arista que culmina la pared. Estas pedreras han contribuído sin duda también a la alimentación del propio glaciar rocoso, aumentando los aportes procedentes de las paredes del circo.

El circo presenta paredes verticales, labradas aprovechando de forma clara la disposición de los estratos de cuarcita y con marcas de canales de gelifracción y canales de avalancha probablemente aún funcionales ${ }^{3}$ que se notan también sobre las pedreras.

Cuadro de parámetros cuantitativos de los glaciares rocosos de la Sierra del Suspirón (León)

\begin{tabular}{|l|c|c|c|c|c|c|c|c|}
\hline $\begin{array}{l}\text { Nombre } \\
\text { de1 }\end{array}$ & $\begin{array}{c}\text { Raíz } \\
(\mathrm{m} .)\end{array}$ & $\begin{array}{c}\text { Frente } \\
(\mathrm{m} .)\end{array}$ & $\begin{array}{c}\text { Cresta } \\
(\mathrm{m} .)\end{array}$ & $\begin{array}{l}\text { Area } \\
\left(\mathrm{Km}^{2}\right)\end{array}$ & $\begin{array}{c}\text { Long } \\
(\mathrm{m} .)\end{array}$ & $\begin{array}{c}\text { Anch } \\
(\mathrm{m} .)\end{array}$ & $\begin{array}{c}\text { Desn. } \\
(\mathrm{m} .)\end{array}$ & $\begin{array}{c}\text { Pend. } \\
(\circ)\end{array}$ \\
\hline $\begin{array}{l}\text { Arcos } \\
\text { del } \\
\text { Agua }\end{array}$ & 1.950 & 1.910 & 2.060 & 0.016 & 120 & 50 & 40 & $19 \circ$ \\
\hline $\begin{array}{l}\text { Peña } \\
\text { Cefera }\end{array}$ & 1.760 & 1.600 & 2.011 & 0.085 & 600 & 120 & 160 & $14 \circ$ \\
\hline
\end{tabular}

El glaciar rocoso posee un recubrimiento vegetal muy pobre, limitado a ejemplares de piorno (Cytisus purgans) que forman rodales aislados y discontínuos que tienden a seguir el dibujo de las ondas de flujo. El carácter de fuerte heterometría, con especial predominio de grandes bloques, que caracteriza la superficie del glaciar rocoso, junto a la ausencia total de elementos finos, hace casi imposible el arraigo de la vegetación, excepción hecha de los líquenes y musgos que tapizan la superficie de las rocas. La extrema facilidad para la percolación del agua, y la existencia de una gran cantidad de huecos intersticiales entre la masa de derrubios, una vez desaparecido el hielo que los empastaba, se deja sentir en el sonido que produce hoy día el agua que circula por el fondo de la masa de rocas, perfectamente audible desde la superficie del glaciar rocoso.

En conclusión, se trata de un glaciar rocoso fósil, del tipo de glaciar rocoso en lengua, constituyendo un ejemplo modélico de esta clase de formas tardiglaciares, adaptado al fondo de una artesa profunda, con perfil en forma de canal (Gómez ORTiz y LloBeT, 1988: 533).

$10 \mathrm{~cm}$. de profundidad labrada sobre una superficie pulida y orientada aguas abajo, es decir, en dirección SE.

${ }^{3}$ La existencia de aludes de nieve en las abruptas vertientes del macizo de Arcos del Agua, al menos hace años, es información oral procedente de los vecinos del próximo pueblo de Fasgar. 
FIG. 3 :

EL GLACIAR ROCOSO DE ARCOS DEL AGUA.
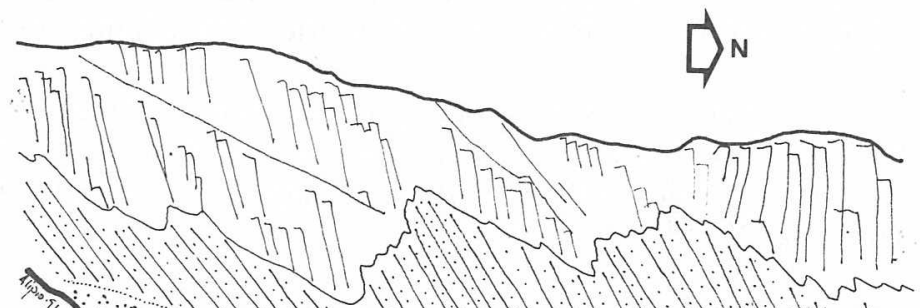

D 115 d an

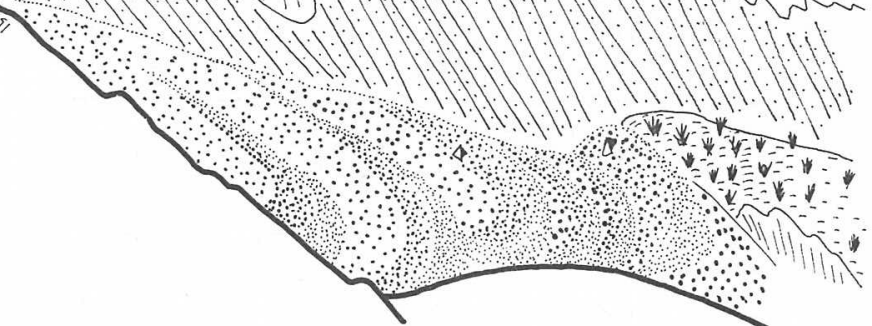

EIG. 4:

EL GLACIAR ROCOSO DE PEÑA CEFERA.

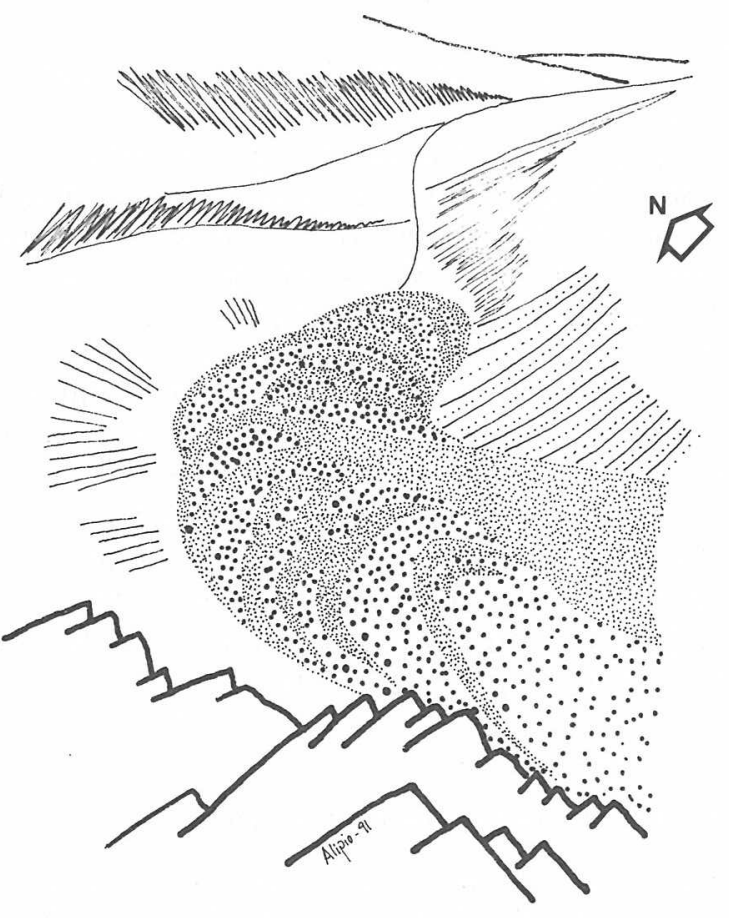




\section{CONCLUSIÓN: LA IMBRICACIÓN DE LAS FORMAS TARDIGLACIARES EN LOS MODELADOS GLACIAR Y PERIGLACIAR DE LA SIERRA}

Sobre la alineación cuarcítica de la Sierra del Suspirón se desarrollo un conjunto de aparatos glaciares de características marginales. Sólamente en el sector culminante de la $S^{\mathrm{a}}$. alcanzaron dimensiones notables, si bien su impronta en el conjunto del paisaje serrano no es en absoluto despreciable, pues introducen matices morfológicos que acentúan el carácter de transición entre montaña media y alta montaña que singulariza a la Sierra del Suspirón.

Enmarcados en el paisaje glaciado del sector culminante de la Sierra, aparecen hoy día unas herencias morfoclimáticas ligadas a la fase tardía de esa morfogénesis glaciar que se concretan en una morrena de nevero y dos glaciares rocosos. Se trata de formas fósiles cuya génesis, excepto para la morrena de nevero, resulta hoy ya muy difícil de determinar, pues las evidencias existentes apenas permiten dilucidar si se trató de glaciares rocosos con núcleo de hielo o de glaciares rocosos con hielo intersticial.

Por su disposición, nos inclinamos a pensar que se trata de glaciares rocosos del primer tipo, pues ambos de desarrollan en las áreas más sombreadas de los primitivos circos y artesas, donde hasta fechas más tardías permanecerían las últimas manchas de hielo glaciar; $\mathrm{Si}$, por el contrario, su génesis hubiera estado ligada a la formación de hielo intersticial en el material de los canchales, se habrían desarrollado también en otras áreas de los circos, donde el material de gelifracción es tanto o más abundante, y eso no ocurrió. De todas formas nos mostramos de acuerdo con DRAMIS y SMIRAGLIA (1986: 212) en la idea de que esta controversia sobre el origen glacial o periglacial de los glaciares rocosos carece de sentido, puesto que ambos tipos de hielo pudieron funcionar al mismo tiempo en la génesis de este tipo de formas.

Por lo que respecta a su edad, todos los autores consultados (TRICART y CAIlleuX, 1962: 226, GuTIÉRREZ ElorZA y PEÑA MONNE, 1981: 18, DRAMIS y SMIRAGLIA, 1986: 213, GÓMEZ ORTIZ y LlOBET, 1988: 532) coinciden en situarla en un periodo que abarca entre el 15000 y el 9000 BP y que recibe diferentes nombres (tardiglaciar würmiense, Tardoglacial, Dryas Superior, Nueva Tundra, etc.)

El carácter de modelado transicional entre dos sistemas morfoclimáticos diferentes -el glaciar y el periglaciar- que poseen los glaciares rocosos y las guirnaldas o morrenas de nevero es un hecho resaltado por todos los autores que han estudiado estas formas del relieve (DRAMIS y SMIRAGLIA, 1986: 211).

En los ejemplos presentes en la Sierra del Suspirón este carácter destaca especialmente, puesto que las formas tardiglaciares se enmarcan en ámbitos geomorfológicos más amplios que deben los caracteres más notables de su morfología a la dinámica glaciar. En efecto, ocupan parte de la superficie de circos y artesas glaciares que albergaron con anterioridad masas de hielo 


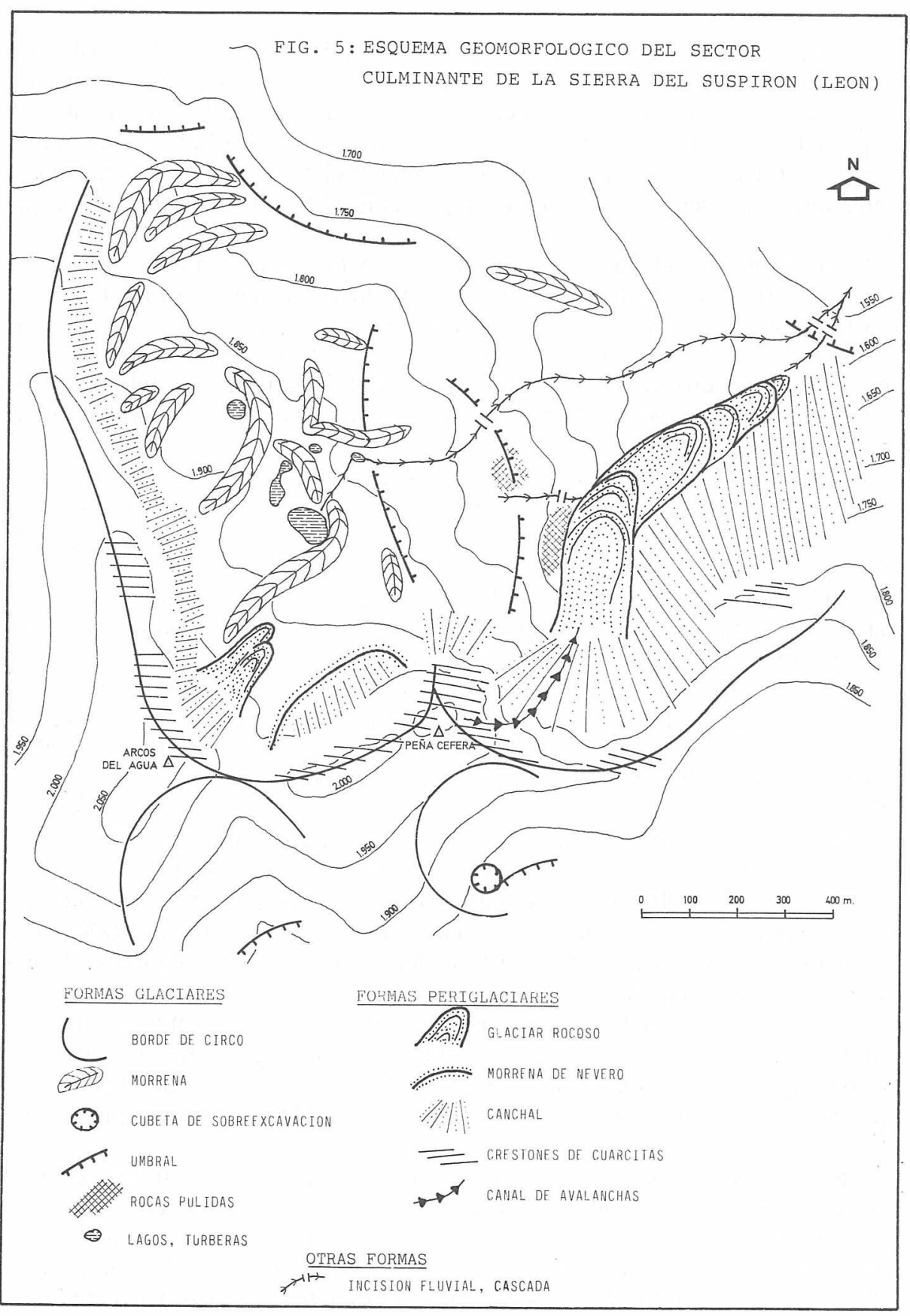


muy voluminosas y con una capacidad morfogenética atestiguada en los depósitos morrénicos situados en cotas más bajas que los propios glaciares rocosos.

Por otro lado, los modelados tardiglaciares, especialmente los de menor tamaño como las morrenas de nevero, aparecen disimulados y en parte fosilizados por los derrubios originados en un sistema morfoclimático en parte contemporáneo y en parte posterior a ellos en el tiempo, el sistema periglaciar (BOYE, 1952: 27).

Sin embargo, sólo han podido desarrollarse bajo condiciones muy específicas de litología, estructura y orientación, pues, a diferencia de las formas estrictamente glaciares y estrictamente periglaciares, que poseen un carácter mucho más generalizado, las formas de modelado ligadas a una geomorfogenesis glaciar tardía constituyen formas singulares, únicas y aisladas, imbricadas a modo de nexo de unión entre las anteriores, de tal forma que constituyen una suerte de excepcionales testimonios del gradual paso entre dos sistemas morfoclimáticos diferentes que se suceden en las montañas cantábricas a lo largo del Cuaternario. Pero además su significado climático va más allá, puesto que en las zonas en las que aparezcan con suficiente abundancia pueden servir de base para efectuar la reconstrucción climática de este período tardoglacial (DRAMIS y SMIRAGLIA, 1986: 213), especialmente en lo que se refiere a la existencia de un permafrost discontinuo y la extensión del mismo.

Su carácter excepcional les confiere, pues, un valor geomorfológico muy elevado, por lo que su presencia complica y enriquece el paisaje de la alta montaña cantábrica.

\section{BIBLIOGRAFIA}

BOYE, M. (1952): «Névés et érosion glaciaire». Revue de Géomorphologie Dynamique, III, pp. 20-36.

DRAMIS, F. y SMIRAGLIA, C. (1986): «I rock-glaciers. Problemi e meto di di studio. Rassegna bibliografica». Rivista Geografica Italiana, $\mathrm{n}^{2} .93$, pp. 209-228.

GOMEZ ORTIZ, A. y LLOBET, S. (1988): «Geomorfogénesis periglacial en Sierra Nevada: formas heredadas y modelados actuales en la unidad geográfica del Mulhacén». Estudios Geograficos, t. XLIX, nº. 193, pp. 527-558.

GRUPO NAZIONALE GEOGRAFIA FISICA E GEOMORFOLOGIA, (1987): «Nuovi dati per lo studio dei rock-glaciers del gruppo OrtlesCevedale (Alpi)». Rivista Geografica Italiana, ํㅡ. 94, pp. 425-450.

GUTIÉRREZ ELORZA, M. y PEÑA MONNE, J.L. (1981): «Los glaciares rocosos y el modelado acompañante en el área de la Bonaigua (Pirineo de Lérida)». Boletín Geológico y Minero, t. XCII-II, pp. 101-110.

SMIRAGLIA, C. (1985): «Contributo alla conoscenza dei rock-glaciers delle Alpi Italiane. I rock-glaciers del Monte Confinale (Alta Valtellina)». 
Rivista Geografica Italiana, $\mathrm{n}^{\circ}$. 92, pp. 117-140.

TRICART, J. y CAILLEUX, A. (1962): Traité de Geomorfologie. Vol. III. Le modelé glaciaire et nival. París: SEDES.

RESUMEN: En el sector culminante de la Sierra del Suspirón (borde Sur de la Cordillera Cantábrica, provincia de León) se desarrollaron, en la fase final del proceso de retroceso glaciar, una serie de formas de modelado características de la transición entre los sistemas morfoclimáticos glaciar y periglaciar: una morrena de nevero y dos glaciares rocosos. Se analizan sus características y dimensiones, se determina su origen y se establece su relación con las formas glaciares y periglaciares entre las que se imbrican. Se ofrece una representación gráfica y cartográfica de las mismas.

PALABRAS CLAVE: Glaciares rocosos, morrenas de nevero, glaciar, periglaciar, tardiglaciar.

RÉSUMÉ: Dans le secteur culminant de la Sierra du Suspirón (sur le versant Sud de la Cordillère Cantabrique, province de León) se sont développés, dans l'ultime étape du recul du glacier, une série de formes d'un modelage caractéristique de la transition entre les systèmes morphoclimatiques glaciaire et périglaciaire: une moraine de névé et deux glaciers rocheux. Nous analyserons leurs caractéristiques et dimensions, nous préciserons leur origine et nous établirons leur relation avec les formes glacières et périglaciaires parmi lesquelles ils s'imbriquent. Nous offrirons une représentation graphique et cartographique de ces glaciaires. MOTS CLÉ: Glaciers rocheux, moraines de névé, glaciaire, periglaciaire, tardiglaciaire.

SUMMARY: In the highest sector of the Sierra del Suspirón (the southern edge of the Cantabrian Highlands, province of León) there are, in the final phase of the process of glacial retrocession, a series of shaped forms which are characteristic of the transition between the glacial and periglacial morphoclimatic systems: nivomoraine and two rock glaciers. Their characteristics and dimensions are analysed, their origin is determined and their relation to the glacial and periglacial forms among which they stand is established. Graphic and cartographic details are included.

KEY WORDS: Rock glaciers, nivomoraines, glacial, periglacial, tardiglacial. 